\title{
Some cyclic solutions to the three table Oberwolfach problem
}

\author{
M. A. Ollis \\ Marlboro College, South Road, Marlboro, VT 05344, USA \\ matt@marlboro.edu \\ Submitted: Jun 2, 2005; Accepted: Nov 9, 2005; Published: Nov 15, 2005 \\ Mathematics Subject Classification: 05C70
}

\begin{abstract}
We use graceful labellings of paths to give a new way of constructing terraces for cyclic groups. These terraces are then used to find cyclic solutions to the three table Oberwolfach problem, $\operatorname{OP}(r, r, s)$, where two of the tables have equal size. In particular we show that, for every odd $r \geq 3$ and even $r$ with $4 \leq r \leq 16$, there is a number $N_{r}$ such that there is a cyclic solution to $\operatorname{OP}(r, r, s)$ whenever $s \geq N_{r}$. The terraces we are able to construct also prove a conjecture of Anderson: For all $m \geq 3$, there is a terrace of $\mathbb{Z}_{2 m}$ which begins $0,2 k, k, \ldots$ for some $k$.
\end{abstract}

\section{Introduction}

The Oberwolfach problem [6] is to decompose the complete graph $K_{v}$, for odd $v$, into mutually isomorphic 2-factors. If the lengths of the cycles in each 2-factor are $r_{1}, r_{2}, \ldots, r_{t}$ (where $r_{1}+r_{2} \cdots+r_{t}=v$ ) then the problem is denoted $\mathrm{OP}\left(r_{1}, r_{2}, \ldots, r_{t}\right)$.

Label the vertices of $K_{v}$ with the symbols of $\mathbb{Z}_{v-1} \cup\{\infty\}$, and let $x+\infty=\infty$ for $x \in \mathbb{Z}_{v-1}$. A solution to $\mathrm{OP}\left(r_{1}, r_{2}, \ldots, r_{t}\right)$ is called cyclic if $x+\mathcal{F}$ is a 2 -factor whenever $\mathcal{F}$ is a 2 -factor and $x \in \mathbb{Z}_{v-1}$.

The goal of this paper is to find cyclic solutions for $\mathrm{OP}(r, r, s)$. Hilton and Johnson [9] have shown that $\mathrm{OP}(r, r, s)$ has a solution for all odd $s$ when $r \in\{3,4\}$, excluding the insoluble case $\mathrm{OP}(3,3,5)$, and for all odd $s \geq 12 r-1$ when $r \geq 5$. These solutions are not, in general, cyclic.

In this paper we show that for every odd $r \geq 3$ and even $r$ with $4 \leq r \leq 16$ there is a number $N_{r}$ such that $\operatorname{OP}(r, r, s)$ has a cyclic solution whenever $s \geq N_{r}$. Our value for $N_{r}$ is less than $12 r-1$, so many of our solutions are to previously unsolved problems. In particular, we show that $\mathrm{OP}(r, r, s)$ has a cyclic solution for every odd $s \geq 5$ when $3 \leq r \leq 13$, excluding $\mathrm{OP}(3,3,5)$.

We now define terraces and graceful labellings - the two concepts that allow us to find the solutions. 
Let $G$ be a group of order $n$ and $\mathbf{a}=\left(a_{1}, a_{2}, \ldots, a_{n}\right)$ an arrangement of the elements of $G$. Define $\mathbf{b}=\left(b_{1}, b_{2}, \ldots, b_{n-1}\right)$, where $b_{i}=a_{i}^{-1} a_{i+1}$. If $\mathbf{b}$ contains each involution of $G$ exactly once and two occurrences (not necessarily distinct) from each set $\left\{g, g^{-1}: g^{2} \neq e\right\}$, then $\mathbf{a}$ is called a terrace for $G$ and $\mathbf{b}$ is the associated 2-sequencing. Terraces were introduced in [3] to construct quasi-complete Latin squares. Elementary abelian 2-groups of order at least 4 do not have terraces-Bailey's Conjecture [3] is that these are the only groups without terraces, see [11] for more details.

Let $T$ be a tree on $n$ vertices. Label the vertices of $T$ with the integers $1,2, \ldots, n$. The labelling is called graceful if

$$
\{|u-v|: u v \text { is an edge }\}=\{1,2, \ldots, n-1\} .
$$

The Ringel-Kotzig graceful tree conjecture is that all trees have a graceful labelling, see [5] for the current state of knowledge.

\section{Terraces from Graceful Labellings}

For the remainder of the paper we consider only terraces for the additive cyclic groups $\mathbb{Z}_{n}$ (arithmetic modulo $n$ ) and graceful labellings of paths $P_{n}$.

Given a terrace $\mathbf{a}$ of $\mathbb{Z}_{n}$ then the translate $x+\mathbf{a}$ is a terrace, and has the same 2sequencing [3]. If the first element of a terrace is 0 , then the terrace is called basic. Every terrace may be translated to a basic terrace.

Example 2.1 The sequence $(0,1, n-1,2, n-2, \ldots)$ is a basic terrace for $\mathbb{Z}_{n}[10,13]$, called the Lucas-Walecki-Williams terrace or LWW terrace. The associated 2-sequencing is $(1, n-2,3, n-4,5, \ldots)$.

There is an easy way to get from a graceful labelling of $P_{n}$, the path of length $n$, to a terrace for the cyclic group $\mathbb{Z}_{n}$. Simply take the list of integers from the graceful labelling, and consider them modulo $n$. The only change in symbols is that $n$ becomes 0 . The differences in the graceful labelling are exactly one of $\pm i$ (as integers) for each integer $i$ in the range $1 \leq i \leq n-1$. This satisfies the requirements of a terrace (when taken modulo $n)$. For example, the graceful labelling $(n, 1, n-1,2, n-2, \ldots)$ becomes the LWW terrace.

For $\mathbb{Z}_{n}$, if $\mathbf{a}$ is a terrace, then $-\mathbf{a}$ and the reverse of $\mathbf{a}$ are also terraces [3]. Similarly for $P_{n}$, the reverse of a graceful labelling and the complementary labelling which replaces $i$ with $n-i$ are also graceful.

A graceful labelling is called $y$-pendant if one of the pendant vertices is labelled $y$. The following result gives a new construction of terraces.

Theorem 2.1 Suppose that $P_{m}$ and $P_{n}$ have y-pendant graceful labellings. Then $\mathbb{Z}_{m+n}$ has a terrace. 
Proof: Suppose that the labelling of $P_{m}$ ends with $y$ and that the labelling of $P_{n}$ starts with $y$ (this can be arranged by taking the reverses of given labellings if required), say $\mathbf{g}=\left(g_{1}, g_{2}, \ldots g_{m}\right)$ and $\mathbf{h}=\left(h_{1}, h_{2}, \ldots, h_{n}\right)$ where $g_{m}=y=h_{1}$.

Let $\mathbf{a}=\left(a_{1}, a_{2}, \ldots, a_{m+n}\right)$, where

$$
a_{i}= \begin{cases}g_{i} & \text { if } i \leq m \\ h_{i-m}+m & \text { if } i>m\end{cases}
$$

The sequence a contains each of the numbers from 1 to $m+n$. Considering the elements of $\mathbf{a}$ as integers, we have the differences $\pm i$ for $1 \leq i \leq m-1$ (as $\mathbf{g}$ is graceful), followed by $m$, and then the differences $\pm i$ for $1 \leq i \leq n-1$ (as $\mathbf{h}$ is graceful). Taken modulo $m+n$, this last is equivalent to $\pm i$ for $m+1 \leq m+n-1$. Altogether we have the differences $\pm i$ for $1 \leq i \leq m+n-1$ and so $\mathbf{a}$ is a terrace for $\mathbb{Z}_{m+n}$.

The following result answers the existence question for $y$-pendant graceful labellings of paths.

Theorem $2.2[4,7]$ There is a y-pendant graceful labelling of $P_{n}$ for every $y \leq n$.

In [1], Anderson conjectured that for all even $n$ the cyclic group $\mathbb{Z}_{n}$ has a terrace which begins $0,2 k, k, \ldots$ for some $k \in \mathbb{Z}_{n}$. It was shown that the truth of the conjecture implies the existence of terraces for all dihedral groups. Later work [2] gave more complicated constructions and proved that all dihedral groups have terraces without needing to prove the conjecture. Theorem 2.3 implies that the conjecture is true, and hence the original constructions are sufficient.

Theorem 2.3 There is a terrace which begins $0,2,1, \ldots$ for $\mathbb{Z}_{n}$ if and only if $n \geq 3$ and $n \neq 4$.

Proof: Clearly there is no terrace of the required form for $n \in\{1,2\}$ and it is easy to check that there is no such terrace for $\mathbb{Z}_{4}$. For $\mathbb{Z}_{3}$, we have the terrace $(0,2,1)$.

Now consider $n \geq 5$. Let $\mathbf{g}$ be the 2-pendant graceful labelling $(1,3,2)$ of $P_{3}$ and let $\mathbf{h}$ be a 2-pendant graceful labelling of $P_{n-3}$ (such a labelling exists for all $n \geq 5$ by Theorem 2.2).

Apply Theorem 2.1 to $\mathbf{g}$ and $\mathbf{h}$ with $y=2$. We obtain a terrace for $\mathbb{Z}_{n}$ of the form $(1,3,2,5, \ldots)$. The translate $(n-1)+(1,3,2,5, \ldots)$ is then $(0,2,1,4, \ldots)$.

\section{The Oberwolfach Problem}

Before stating Theorem 3.1, which links terraces to the Oberwolfach Problem, we need some more definitions.

Let $\mathbf{a}=\left(a_{1}, a_{2}, \ldots, a_{n}\right)$ be a basic terrace for $\mathbb{Z}_{n}$ with 2 -sequencing $\mathbf{b}=\left(b_{1}, b_{2}, \ldots, b_{n-1}\right)$. If $b_{r}=a_{r}$ then $r$ is a left match-point of $\mathbf{b}$. A left match-point is right-flexible if at least one of the following is true. 
- $n$ is even and $\frac{n}{2}$ occurs somewhere to the right of $b_{r}$ in $\mathbf{b}$

- There is an element $x$ with $\pm x$ occuring to both somewhere to the left and somewhere to the right of $b_{r}$ in $\mathbf{b}$.

There are also the notions of right match-points and left-flexibility [12], but we do not need them here.

Theorem 3.1 [12] If $\mathbb{Z}_{n}$ has a 2-sequencing with a right-flexible left match-point $r$ then there is a cyclic solution to $\mathrm{OP}(r, r, s)$, where $s=2(n-r)+1$.

The construction which proves this theorem involves the "lifting" of a 2-sequencing to a "symmetric sequencing" for the cyclic group of twice the size and then showing that the associated terrace of this is a "symmetrically sectionable directed terrace." The details can be found in [12].

We now need to be able to construct terraces with left match-points (their rightflexibility will be considered later).

Theorem 3.2 Let $\mathbf{g}=\left(g_{1}, g_{2}, \ldots, g_{m}\right)$ be a graceful labelling of $P_{m}$ with $g_{r}-g_{1}=g_{r+1}-g_{r}$, for some $r$. Then $\mathbb{Z}_{m+n}$ has a basic terrace with $r$ as a left match-point of the associated 2-sequencing for $n=0$ or $n \geq g_{m}$.

Proof: If $n=0$, consider the elements of $\mathbf{g}$ modulo $n$ and let $\mathbf{a}$ be the translate which is basic. Then the 2 -sequencing of a has $r$ as a left match-point.

Now consider a fixed $n \geq g_{m}$. Let $\mathbf{h}$ be a $g_{m}$-pendant graceful labelling of $P_{n}$ (this exists by Theorem 2.2). Apply Theorem 2.1 to $\mathbf{g}$ and $\mathbf{h}$ to obtain a terrace for $\mathbb{Z}_{m+n}$. Again, letting a be the translate which is basic, we get that the associated 2-sequencing has $r$ as a left match-point.

We are now in position to prove our main result.

Theorem 3.3 Let $r=2 k+1$ and set $N_{r}=2\left\lfloor\frac{3 k+1}{2}\right\rfloor+2 k+3$. There is a cyclic solution to $\mathrm{OP}(r, r, s)$ whenever $s \geq N_{r}$.

Proof: Let $\mathbf{g}$ be the complementary labelling to the graceful labelling for $P_{m}$ which gives the LWW terrace:

$$
\mathbf{g}=\left(1, n, 2, n-1,3, n-2, \ldots,\left\lfloor\frac{m}{2}\right\rfloor+1\right)
$$

If $m=3 k+1$ then we have $g_{2 k+1}=k+1$ and $g_{2 k+2}=2 k+1$. Hence

$$
g_{2 k+2}-g_{2 k+1}=k=g_{2 k+1}-g_{1} .
$$

Take $n \geq\left\lfloor\frac{m}{2}\right\rfloor+1$. By Theorem 3.2 we can construct a basic terrace whose 2-sequencing b has $2 k+1$ as a left match-point. This left match-point is right flexible as $-(k+1)$ occurs in position $2 k$ and $\pm(k+1)$ occurs somewhere after position $m$.

Applying Theorem 3.1 now gives the result. 
To prove an analogous result for even $r$ it would suffice to give a graceful labelling $\left(g_{1}, g_{2}, \ldots g_{m}\right)$ of $P_{m}$ with $g_{r+1}-g_{r}=g_{r}-g_{1}$, and to check that the required right-flexibility property holds.

Table 1 gives some graceful labellings of $P_{m}$ for which $g_{r+1}-g_{r}=g_{r}-g_{1}$. As we have all even values of $r$ in the range $4 \leq r \leq 16$, we have the result that there is a number $N_{r}$ such that a cyclic solution to $\mathrm{OP}(r, r, s)$ exists whenever $s \geq N_{r}$ for these values of $r$. The table also includes some odd values of $r$ for which the value of $N_{r}$ improves on that of Theorem 3.3. Right-flexibility in the appropriate 2-sequencing is straightforward to confirm.

Table 1: Some graceful labellings

\begin{tabular}{r|r|l|r}
$r$ & $m$ & A graceful labelling of $P_{m}$ & $N_{r}$ \\
\hline 4 & 5 & $(2,5,1,3,4)$ & 11 \\
5 & 7 & $(3,7,1,6,4,5,2)$ & 9 \\
6 & 8 & $(4,7,1,8,3,5,6,2)$ & 9 \\
7 & 9 & $(5,2,8,1,9,4,6,7,3)$ & 11 \\
8 & 10 & $(4,7,1,10,2,9,5,6,8,3)$ & 11 \\
9 & 11 & $(5,4,10,2,11,1,8,3,7,9,6)$ & 17 \\
10 & 12 & $(3,10,4,12,1,11,2,7,8,6,9,5)$ & 15 \\
11 & 13 & $(8,3,11,5,7,4,13,1,12,2,9,10,6)$ & 17 \\
12 & 14 & $(2,12,3,14,1,13,5,11,4,9,8,6,10,7)$ & 19 \\
13 & 15 & $(4,11,3,13,2,14,1,15,6,12,8,9,7,10,5)$ & 15 \\
14 & 16 & $(8,9,5,15,3,14,1,16,2,11,6,13,7,10,12,4)$ & 13 \\
16 & 17 & $(9,5,14,4,7,12,6,13,2,15,3,17,1,16,8,10,11)$ & 25
\end{tabular}

To conclude, we collect together the old and new results on cyclic solutions to $\mathrm{OP}(r, r, s)$ for small values of $r$.

Theorem 3.4 Except for the insoluble case $\mathrm{OP}(3,3,5)$, there is a cyclic solution to $\mathrm{OP}(r, r, s)$ for all odd $s \geq 5$ and each $r$ in the range $3 \leq r \leq 13$.

Proof: For $r=3$, Theorem 3.3 gives $N_{r}=9$ and [12, Theorem 12] covers the case $s=7$.

Consider $r$ in the range $4 \leq r \leq 8$. Table 2 of [12] gives cyclic solutions for all $s$ in the range $5 \leq s \leq N_{r}$, where $N_{r}$ is taken from Table 1 .

For $r$ in the range $9 \leq r \leq 13$, Table 2 of [12] gives cyclic solutions for $5 \leq s \leq 31-2 r$. Comparing with our Table 1 , this leaves 14 outstanding cases. Of these, $\operatorname{OP}(11,11,11)$ and $\mathrm{OP}(13,13,13)$ are covered in $[8]$ and $\mathrm{OP}(12,12,13)$ by the comments on p. 408 of [12]. The remaining 11 cases can be solved by applying Theorem 3.1 to the terraces (whose 2 -sequencings have right-flexible left match-points $r$ ) in Table 2. Several of these terraces were found using the techniques and examples of [12] — where this is the case we indicate the notation which describes them in that paper. 
Table 2: Some terraces for $\mathbb{Z}_{m}$

\begin{tabular}{r|r|l|r|l}
$r$ & $m$ & A terrace for $\mathbb{Z}_{m}$ & $s$ & Notation \\
\hline 9 & 16 & $(0,14,5,9,4,11,3,13,1,2,15,10,7,8,6,12)$ & 15 & - \\
10 & 16 & $(0,1,3,13,2,6,15,12,4,7,14,10,5,11,9,8)$ & 13 & $T_{1}(8)^{\uparrow}$ \\
11 & 17 & $(0,8,9,14,5,3,1,7,11,4,16,15,12,2,13,10,6)$ & 13 & - \\
& 18 & $(0,4,11,15,9,1,16,17,14,7,5,10,2,3,12,6,8,13)$ & 15 & - \\
12 & 16 & $(0,3,5,4,13,15,11,1,6,10,2,7,14,8,9,12)$ & 9 & - \\
& 17 & $(0,13,10,8,7,2,14,4,6,3,12,16,15,9,1,11,5)$ & 11 & $\operatorname{rev}\left(\Delta_{12}^{1}(17)\right)$ \\
& 19 & $(0,1,3,6,10,15,2,9,17,7,14,16,13,5,11,12,8,18,4)$ & 15 & $\Delta_{14}(19)$ \\
& 20 & $(0,3,11,12,16,7,5,18,4,9,19,14,8,15,17,6,2,1,13,10)$ & 17 & $T_{2}(10)^{\uparrow}$ \\
13 & 16 & $(0,10,8,11,6,4,3,12,15,7,1,5,9,2,13,14)$ & 7 & - \\
& 17 & $(0,1,3,6,10,15,4,11,2,9,13,14,12,7,16,5,8)$ & 9 & $\Delta_{14}(17)$ \\
& 18 & $(0,5,7,9,15,14,6,17,12,16,13,10,4,8,1,2,11,3)$ & 11 & -
\end{tabular}

\section{References}

[1] B.A. Anderson. Sequencings of dicyclic groups II. J. Combin. Math. Combin. Comp., 3:5-27, 1988.

[2] B. A. Anderson. All dicyclic groups of order at least 12 have symmetric sequencings. Contemp. Math., 111:5-21, 1990.

[3] R. A. Bailey. Quasi-complete latin squares: construction and randomization. J. Royal Statist. Soc. Ser. B, 46:323-334, 1984.

[4] E. Flandrin, I. Fournier, and A. Germa. Numérotations gracieuses des chemins [graceful enumerations of paths]. Ars. Combin., 16:149-181, 1983.

[5] J. A. Gallian. A dynamic survey of graph labellings. Electron. J. Combin., Dynamic Surveys(6):95pp, 2001.

[6] R. K. Guy. Unsolved combinatorial problems. In D. J. A. Walsh, editor, Combinatorial mathematics and its applications (Proc. Conf. Oxford 1967), page 121, New York, 1976. Academic Press.

[7] P. Gvozdjak. On the Oberwolfach Problem for Cycles with Multiple Lengths (PhD thesis). Simon Fraser University, 2004.

[8] P. Hell, A. Kotzig, and A. Rosa. Some results on the Oberwolfach problem. Aequationes Math., 12:1-5, 1975.

[9] A. J. W. Hilton and M. Johnson. Some results on the Oberwolfach problem. J. London Math. Soc., 64:513-522, 2001.

[10] E. Lucas. Récréations Mathématiques, Tôme II. Albert Blanchard, Paris, 1892. reprinted 1975. 
[11] M. A. Ollis. Sequenceable groups and related topics. Electron. J. Combin., Dynamic Surveys(10):34pp, 2002.

[12] M. A. Ollis and D. A. Preece. Sectionable terraces and the (generalised) Oberwolfach problem. Discrete Math., 266:399-416, 2003.

[13] E. J. Williams. Experimental designs balanced for the estimation of residual effects of treatments. Aust. J. Scient. Res. A, 2:149-168, 1949. 